\title{
Application of DOE in confocal microscopy for surface measurement
}

\section{Zheng Li, Miro Taphanel, Thomas Längle, Jürgen Beyerer}

Zheng Li, Miro Taphanel, Thomas Längle, Jürgen Beyerer, "Application of DOE in confocal microscopy for surface measurement," Proc. SPIE 11144, Photonics and Education in Measurement Science 2019, 1114411 (17 September 2019); doi: 10.1117/12.2531610

SPIE Event: Joint TC1 - TC2 International Symposium on Photonics and Education in Measurement Science 2019, 2019, Jena, Germany 


\title{
Application of DOE in confocal microscopy for surface measurement
}

\author{
Zheng Li*a,b, Miro Taphanel ${ }^{\mathrm{a}}$, Thomas Längle ${ }^{\mathrm{a}}$, and Jürgen Beyerer ${ }^{\mathrm{a}, \mathrm{b}}$ \\ ${ }^{a}$ Fraunhofer Institute of Optronics, System Technologies and Image Exploitation IOSB, \\ Fraunhoferstr. 1, 76131 Karlsruhe, Germany

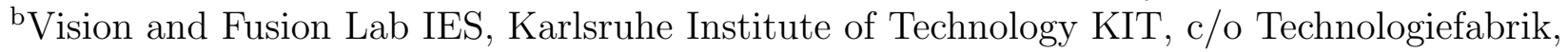 \\ Haid-und-Neu-Str. 7, 76131 Karlsruhe, Germany
}

\begin{abstract}
In traditional confocal microscopy, there is a trade-off between spatial resolution and field of view due to the limitations of objectives. To solve this problem, diffractive optical elements (DOEs) with overlapping apertures are used to generate high-NA illumination spots in a large area. However, currently such DOEs can only be used as illuminators which are not suitable for $3 \mathrm{D}$ surface measurements. In this work, the idea of superposition is utilized to expand the scope of application of the DOEs. These DOEs are designed by simulation and tested in the experiments. The results show that the proposed DOEs can be used in 3D surface measurements and have the potential to solve the problem of high-NA objectives.
\end{abstract}

Keywords: DOE, diffractive optics, confocal microscopy, surface measurement, numerical aperture

\section{INTRODUCTION}

Confocal microscopy has long become the golden standard in life sciences and other fields ${ }^{1}$. In a single-spot laser scanning confocal microscope, a highly focused spot is produced by an objective to illuminate a tiny part of the object. The bright spot on the object is again imaged by the objective onto the image sensor. A pinhole is used to block out-of-focus light scattered by the object to have a sharper image. In this way, much better axial resolution than wide-field microscopy can be achieved and 3D images of the object can be obtained through layer-by-layer scanning. Furthermore, a multi-spot array like a Nipkow disk can be used to increase the scanning speed.

One of the key components in a confocal microscope is the objective. The resolution of the microscope is directly related to the produced spot size, and the spot size is determined by the numerical aperture (NA) of the objective, which is defined as

$$
\mathrm{NA}=n \sin \theta
$$

where $n$ is the refractive index of the medium in which the objective works and $\theta$ is the half angle of the light cone which the objective can collect.

High-NA objectives provide better resolution. However, high-NA objectives with large field of view, e.g. lithography lenses, are very difficult and expensive to design and produce, which makes them impractical for microscopic applications $^{2}$. The field of view of an objective usually gets smaller with the increase of NA, which means only a small portion of the sample can be imaged and more scanning is required for inspection of a large area. Besides, due to the complex structures of high-NA lenses, they are also very expensive.

In order to solve these problems, using DOEs as array illuminators has been proposed to scan large-area samples with comparable resolution to the standard confocal microscopy ${ }^{3-6}$. They are able to produce high-NA spots over a large area. Furthermore, they can be easily replicated by lithography with a very low cost.

Further author information: (Send correspondence to Zheng Li)

Zheng Li: E-mail: zheng.li@iosb.fraunhofer.de, Telephone: +49 7216091528

Miro Taphanel: E-mail: miro.taphanel@iosb.fraunhoer.de, Telephone: +497216091 389

Photonics and Education in Measurement Science 2019, edited by Maik Rosenberger,

Paul-Gerald Dittrich, Bernhard Zagar, Proc. of SPIE Vol. 11144, 1114411

(C) 2019 SPIE · CCC code: 0277-786X/19/\$21 · doi: 10.1117/12.2531610 


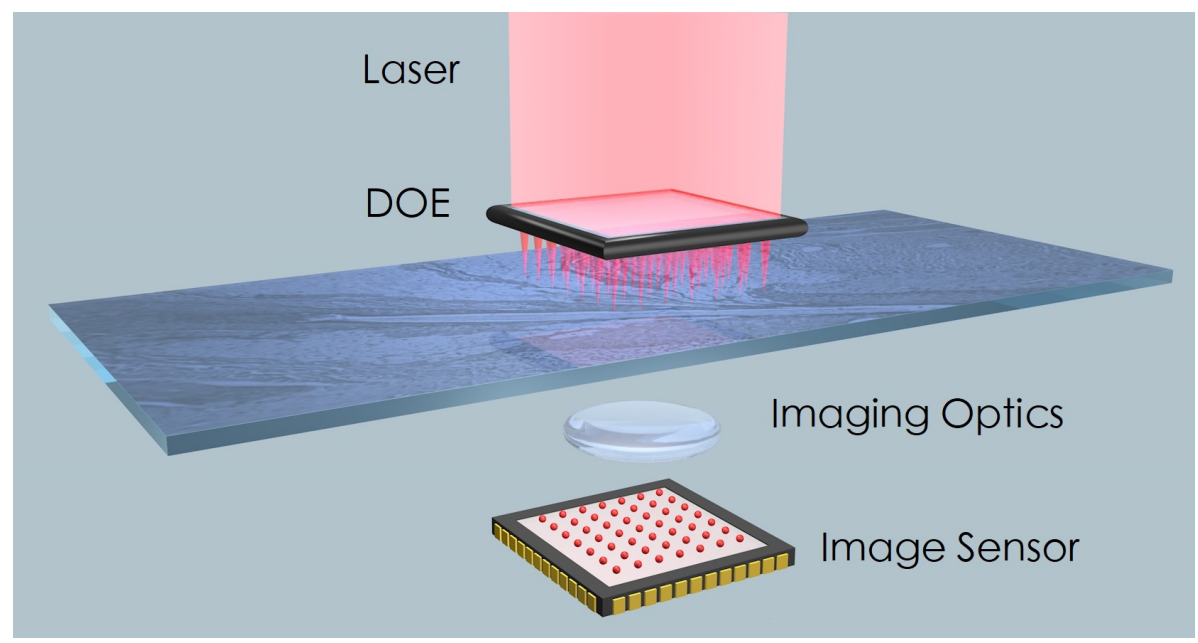

Figure 1. Optical configuration of a multi-spot scanning microscope based on a DOE illuminator ${ }^{3}$.

Figure 1 shows the principle of a multi-spot scanning microscope based on a DOE illuminator ${ }^{3}$. In this case, the DOE acts as an array of Fresnel lenses with overlapping apertures. It focuses plane waves into tiny illumination spots. A complete image of the sample is obtained by scanning with the spot array.

However, current demonstrations only shows their capabilities to be used as illuminators. The imaging systems are placed on the opposite side and only transparent or semi-transparent samples can be measured. When measuring opaque samples, the DOEs have to be put between the sample and imaging system. In such situations, they will cause severe field disturbance when the spots are imaged through the DOEs. Thus the DOEs in current researches are not suitable for 3D surface measurements. In order to avoid this limitation, we propose new kinds of DOEs which utilize superposition of different field distributions to allow more flexible functionality.

\section{DOE DESIGN AND SIMULATION}

\subsection{Design and Simulation Methods}

To use the DOEs to replace high-NA objectives for fast scanning with high resolution, they need to be able to produce dense spot arrays with high-NA. This is achieved by the concept of overlapping aperture, which is explained by the design procedures in figure 2 .

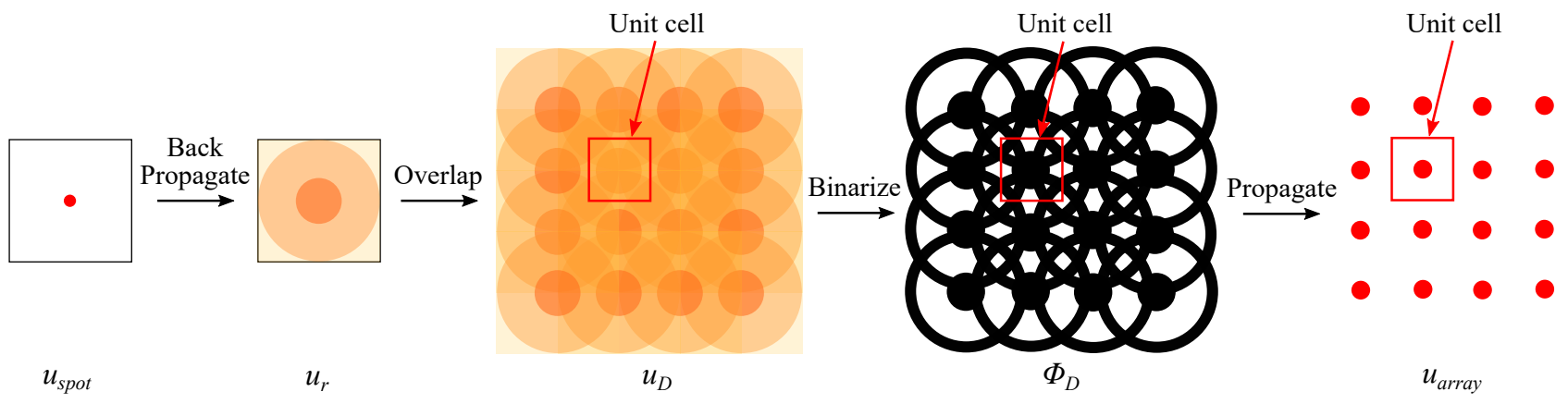

Figure 2. Design procedures for a DOE spot generator with overlapping apertures.

First, the target spot field distribution $u_{\text {spot }}$ is defined. It propagates back and forms a spherical-wave-like field distribution $u_{r}$. Rayleigh-Sommerfeld integral ${ }^{7-9}$ is used as the simulation method for the diffraction field 
propagation, which is shown as

$$
u_{r}=\iint_{\Sigma} u_{\text {spot }}\left(\mathbf{r}^{\prime}\right) \frac{e^{-i k\left|\mathbf{r}-\mathbf{r}^{\prime}\right|} z}{\left|\mathbf{r}-\mathbf{r}^{\prime}\right|^{2}} d x^{\prime} d y^{\prime}
$$

where $\Sigma$ denotes the surface on the boundary, i.e. the plane which $u_{\text {spot }}$ lies in and the semi-infinite sphere behind it, $\mathbf{r}=(x, y, z)$ is the coordinate of $u_{r}, \mathbf{r}^{\prime}=\left(x^{\prime}, y^{\prime}, z^{\prime}\right)$ is the coordinate on $\Sigma$, and $k$ is the wave number. Afterwards, in order to construct a spot array, $u_{r}$ is duplicated and overlapped with a certain pitch to form $u_{D}$. Then the phase of the overlapping field $u_{D}$ is extracted and binarized with a binarization factor $B^{3}$ in the following equation,

$$
\phi_{D}(x, y)=\bmod \left(\left\lfloor\frac{\arg \left[u_{D}(x, y)\right]+B}{\pi}\right\rfloor, 2\right) \pi .
$$

Finally the binarized field propagates back by simulation to verify the spot array which will be actually produced. In this case, the spot is not solely produced by the small unit cell above it, but it will be produced by the original spherical-wave-like field which is already overlapped with the adjacent ones. Every single spot in the array can receive contributions from other unit cells around it. Thus, the NA of the spot is not limited by the pitch anymore. A dense spot array with high NA can be generated in this way.

However, as described in Section 1, such DOEs are not capable of measuring opaque objects, in which case the DOEs and the imaging system must be placed on the same side. To improve that, the idea of superposition is used to add more flexibility to the current DOEs. By simply adding different fields generated from different target light distributions, all the target patterns can be generated with one single DOE ${ }^{10}$. Such kind of DOEs is known as the multi-functional DOEs.

In the following sections, two DOE concepts are designed with this method to overcome the shortcomings of the DOEs in current research for confocal microscopy ${ }^{3-5}$. The new designs have different functions and are both able to measure opaque surfaces.

\subsection{See-through DOE design}

In order to illuminate and image the sample on the same side for confocal surface measurements, the spots need to be seen through the DOEs without severe disturbances.

Figure 3 shows a DOE-based multi-spot scanning confocal microscope for surface measurements. The DOE generates high-NA spots which are imaged by a low-NA objective. In this way, although the low-NA objective will produce larger spots on the image plane, the lateral resolution is still governed by the high-NA illumination spots, which is similar to the principle of super-resolution microscopy like STED ${ }^{11}$ and PALM ${ }^{12}$. Meanwhile, a low-NA lens can offer a large field of view. Thus high resolution and large-area scanning can be achieved at the same time.

However, in the setup above, the original DOEs with overlapping apertures in figure 2 will introduce disturbances during the imaging process. Simulation shows that the spots become irregular or even invisible when they are imaged by the objective and camera ${ }^{13}$. In order to reduce the disturbances added by the DOEs, a plane-wave component is added to form a new field distribution,

$$
u_{D}^{\prime}=u_{D}+W
$$

where $u_{D}$ is the original overlapped field distribution in figure 2 and $W$ is a constant.

In this way, the zero-order diffraction of the DOEs becomes higher. In other words, the DOEs become more transparent. The parameters of the DOEs are optimized iteratively to achieve the highest intensities in the spot centers in the image with the working distance $z=1.095 \mathrm{~mm}$, the plane-wave weight factor $W=13$ and the binarization factor $B=0.41 \pi$ in Eq. (3) for a $11 \times 11$ spot array. The pitch is $100 \mu \mathrm{m}$ and the designed wavelength is $785 \mathrm{~nm}$.

The see-through DOEs can improve the lateral resolution by the high-NA spots produced by the DOE. However, according the the theory of confocal microscopy, such configuration in figure 3 cannot significantly improve the axial resolution when measuring a plane object ${ }^{14}$. The axial resolution will still be strongly restricted by the low-NA objective for surface measurement. 


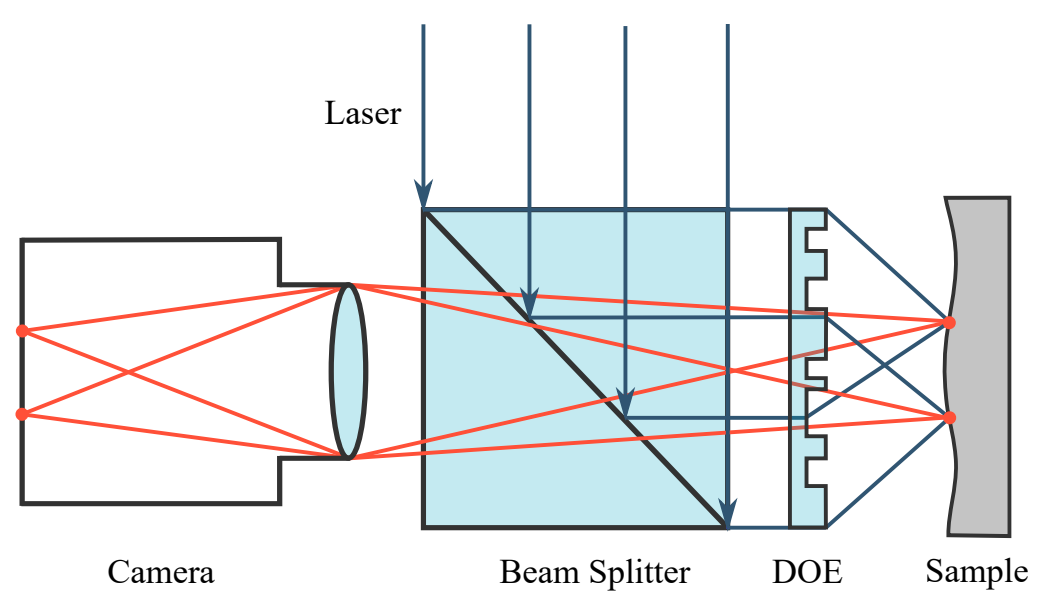

Figure 3. See-through configuration of a DOE-based confocal surface measurement system.

\subsection{Direct-imaging DOE design}

In order to increase the axial resolution and make the DOE suitable for 3D surface measurements, the optical configuration for the direct-imaging DOE design is proposed as figure 4 shows. In this case, the DOE is the superposition of two kinds of lenses with different focal lengths and overlapping apertures. One acts as an illumination lens which converts an incident plane wave into a spot array. The other one is the imaging lenses which acts as part of the imaging optical system. Alternatively, it can also directly image the spots onto a imaging sensor. The two lenses are overlapped as figure 2 shows. In this case, the field distribution $u_{D}^{\prime}$ for the new DOE is calculated by

$$
u_{D}^{\prime}=u_{D}+W u_{l e n s} .
$$

In this way the illumination and the imaging are both high-NA and the system can have the same performance as a high-NA confocal microscope.

The direct-imaging DOEs are also optimized iteratively to achieve the highest intensities in the image spot centers with the working distance $z=1.11 \mathrm{~mm}$, the distance from the DOE to the intermediate image spots $d=21.262 \mathrm{~mm}$, the weight factor $W=0.045$ and the binarization factor $B=0.97 \pi$ in Eq. (3) for a $5 \times 5$ spot array. The pitch is $100 \mu \mathrm{m}$ and the designed wavelength is $785 \mathrm{~nm}$.

The direct-imaging DOEs provide both high lateral and axial resolution. However, simulation shows that irregular interference will be introduced when the number of spots in the array increases. An $11 \times 11$ spot array will already

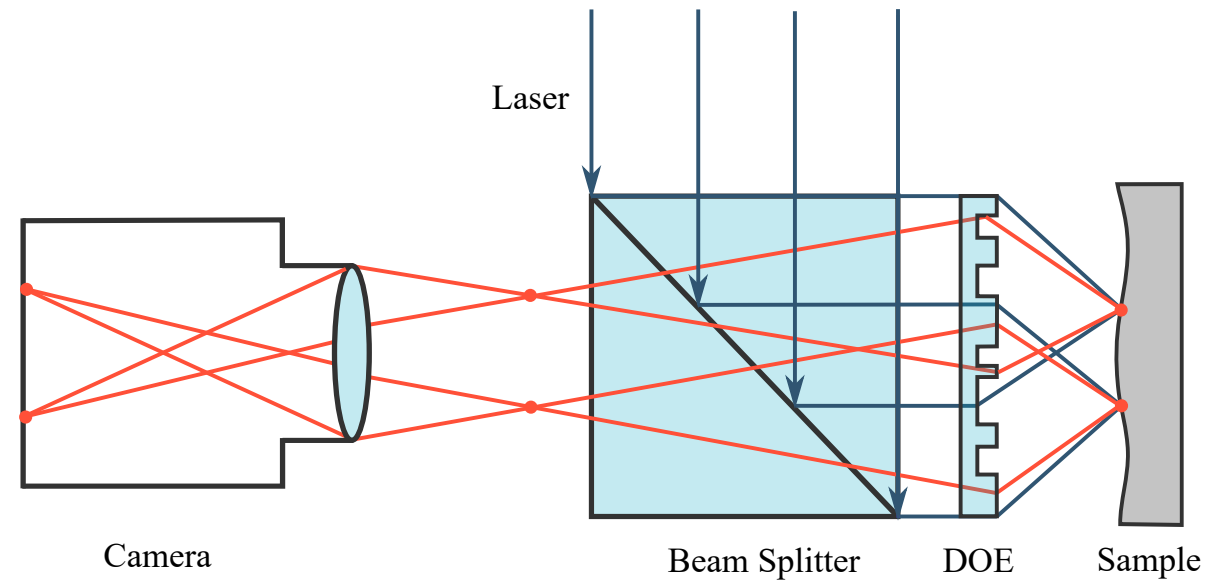

Figure 4. Direct-imaging configuration of a DOE-based confocal surface measurement system. 
make the spots indistinguishable from the background ${ }^{13}$. As mentioned previously, the DOE is composed of two components which are generally two kinds of lenses with different focal lengths. They act as the illumination lenses and imaging lenses respectively. However, they will not work separately as we want for illumination and imaging. They will always take effect at the same time. When projecting the illumination spots, the imaging lenses will also produce a blurred spot around the original one, which is shown in figure 5a. Besides, on the imaging side, due to the overlapping apertures, one spot will not only pass the designed lens to form a spot on the image sensor, but also it will go through the adjacent lenses to form other blurred spots, which is shown in figure 5b. As the number of spots increases, the disturbances will accumulate and the image quality will be reduced.

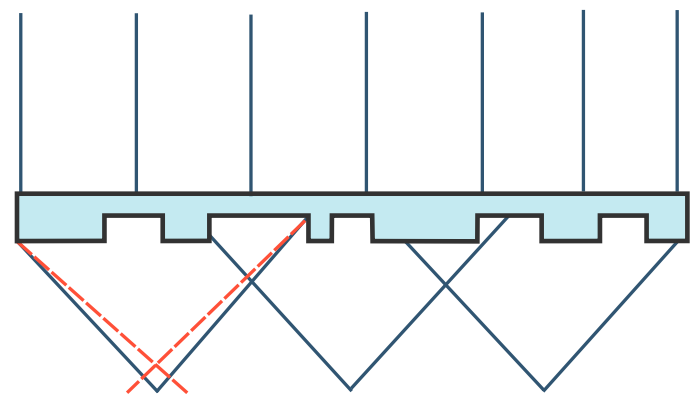

(a) Illumination through the superimposed lens.

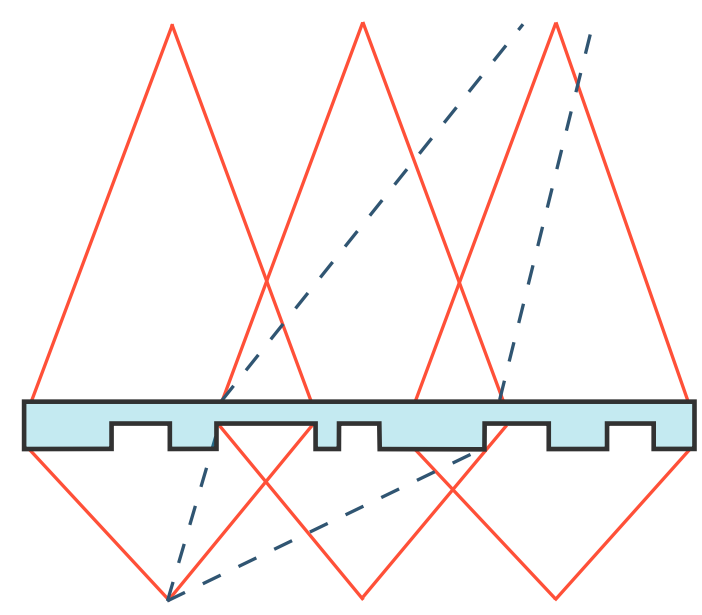

(b) Imaging through the adjacent lens.

Figure 5. Side effects which cause irregular interference patterns for the direct-imaging DOE.

One possible solution is using a line of spots instead of a $2 \mathrm{D}$ grid to reduce the overlapping. In this case, there is no longer interference from all the directions. Furthermore, one spot will receive less disturbances from the spots farther away. Thus the overall disturbances for a single spot can be controlled to an acceptable level and the length of the line can be extended to any desired measurement range.

\section{EXPERIMENT RESULTS}

After the DOEs are designed, a prototype is made by electron-beam lithography in Fraunhofer IOF. The prototype is made of fused silica with a refractive index of $n=1.45$ and has a smallest feature size of $400 \mathrm{~nm}$. The binary structures are etched on the plate with an etching depth of $h=\lambda / 2(n-1)=872 \mathrm{~nm}$ for the designed wavelength $\lambda=785 \mathrm{~nm}$. The following experiments are based on this prototype.

\subsection{See-through DOE Experiments}

Figure $6 \mathrm{c}$ shows the real experiment setup of figure 3. In the setup, a diode laser is coupled into a single mode fiber. It connects to a collimator and produces plane-wave illumination. The light is reflected by the beam splitter and illuminates the DOEs. Underneath there is a CMOS sensor to measure the illumination spots and a mirror to reflect the light. At the top, a camera and an objective is used to image the spots through the DOEs.

In the first experiment, the $11 \times 11$ spot arrays produced by both the original and see-through DOEs are measured by the CMOS sensor from Raspberry Pi Camera V2. Because the $1.12 \mu \mathrm{m}$ pixel size of the sensor is not small enough to resolve the spot laterally, the sensor is moved up and down in $z$ direction to measure the axial point spread function (PSF) of the spots in order to determine the actual NA which has been achieved. The axial PSF measurements for the original and see-through DOEs are shown in figure 6a and 6b respectively.

For the original DOE, the simulated axial full-width at half maximum (FWHM) is $2.3 \mu \mathrm{m}$ and the measured FWHM is $2.67 \mu \mathrm{m}$, which corresponds to an $\mathrm{NA}=0.77^{15,16}$. For the see-through DOE, the simulated FWHM 


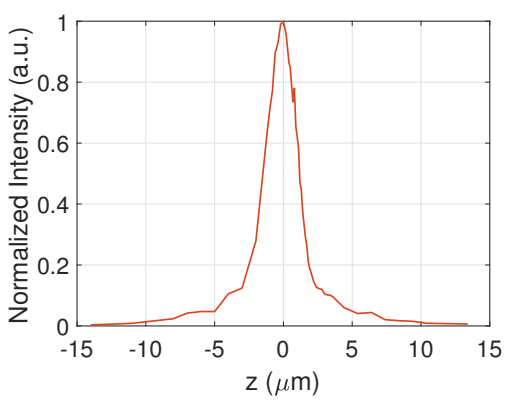

(a) Axial PSF of the original DOE.

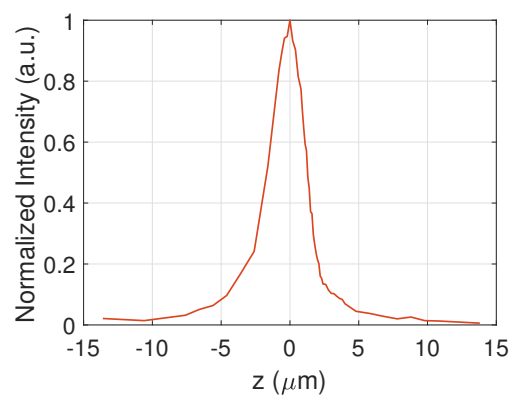

(b) Axial PSF of the see-through DOE.

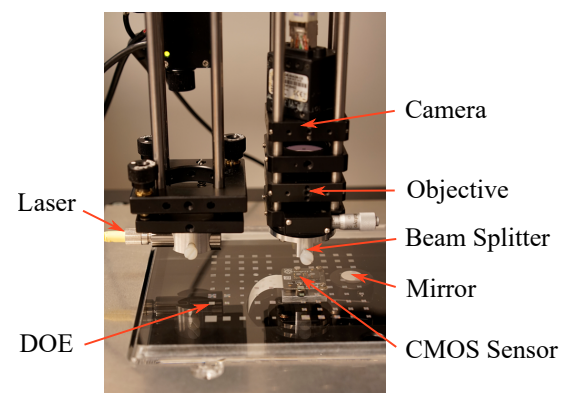

(c) Experiment setup.

Figure 6. Experiments to measure the illumination spots.

is $2.5 \mu \mathrm{m}$ and the measured FWHM is $2.96 \mu \mathrm{m}$, which corresponds to an NA $=0.73$. The experiment results are close to the simulation. The slight deviation can be attributed to that the real diffraction efficiency of the DOEs might be lower and the acceptance angle of the light for the CMOS sensor is limited.

In the second experiment, the illumination spots are reflected by a mirror and they are imaged by the objective and the camera through the DOE. Then the mirror is also moved axially in $z$ direction to measure the confocal axial response. The central spot in the $11 \times 11$ spot array is selected and the axial intensity response in the image by moving the mirror is recorded. The objective has an NA $=0.15$.

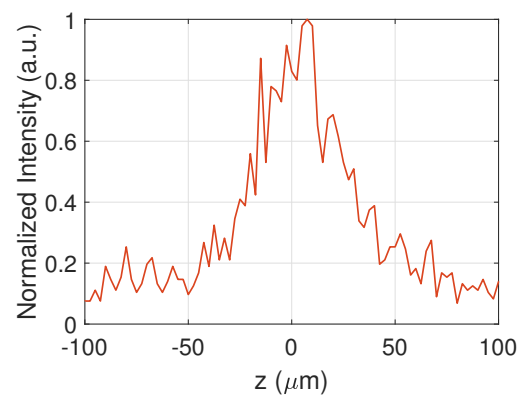

(a) Confocal axial response of the original DOE on a mirror.

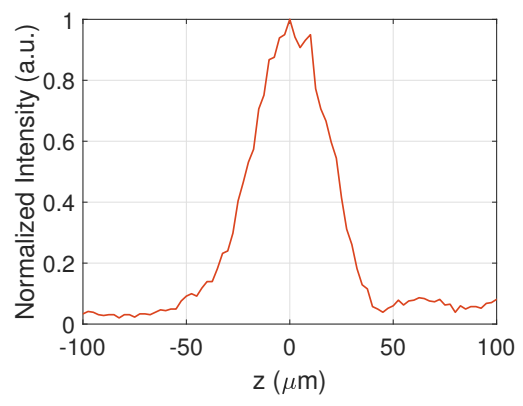

(b) Confocal axial response of the seethrough DOE on a mirror.

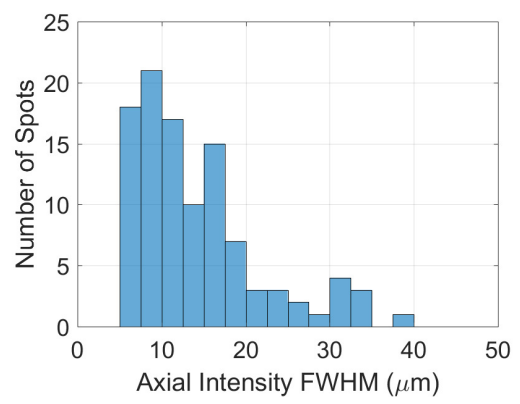

(c) Confocal axial response of the seethrough DOE on a rough surface.

Figure 7. Experiments to measure the confocal axial response.

Figure $7 \mathrm{a}$ and $7 \mathrm{~b}$ show the results of the confocal axial responses for the original DOE and the see-through DOE separately. Obviously, the intensity response of the original DOE is very noisy and its peak is hard to define. Contrarily, the see-through DOE provides a much smoother signal, which proves that it can be fit into the configuration in figure 3 without introducing severe disturbances. It shows a FWHM of $46.2 \mu \mathrm{m}$ which corresponds to an $\mathrm{NA}=0.18$. As predicted by the theory and the simulation, the axial resolution for a plane is only slightly improved and is still highly restricted by the low-NA objective for imaging.

Finally a rough surface on a coin is randomly selected and used as a measurement object. The confocal axial responses of all the spots in the array are recorded. The FWHM of the spots within the measurement range are calculated. The distribution is shown in the histogram in figure 7c. The results show that although still limited by the low-NA objective, a large portion of spots can produce significantly smaller axial FWHM, which increase the measurement sensitivity. The reason is that when the surface is not a perfect horizontal plane, it will be more "point-like". And for a point object, the axial resolution is no longer governed by the low-NA imaging objective in the configuration of figure 3. Detailed mathematical derivation can be found in Ref. 13,14. 


\subsection{Direct-imaging DOE Experiments}

For the direct-imaging DOE, the axial PSF of the produced illumination spot is also measured as figure 8a shows. The measured FWHM is $3.54 \mu \mathrm{m}$ which corresponds to an NA $=0.67$. The produced spots are larger compared to the ones produced by the see-through DOEs because of the contribution from the additional superimposed field as shown in figure 5 a.

After that, a mirror is placed underneath to measure the confocal axial response as figure 4 shows. The results for the central spot of the direct-imaging DOEs in the $5 \times 5$ and $9 \times 9$ spot arrays are shown in figure $8 \mathrm{~b}$ and $8 \mathrm{c}$ respectively. The confocal axial response of the central spot in the $5 \times 5$ array shows a FWHM of $4.57 \mu \mathrm{m}$, which provides much better resolution than the imaging objective with $\mathrm{NA}=0.15$. figure 5 increase with the number of spots, the central spot in the $9 \times 9$ array is completely missing in the irregular interference. Thus a line should be used instead of a grid to avoid such disturbance in real applications.

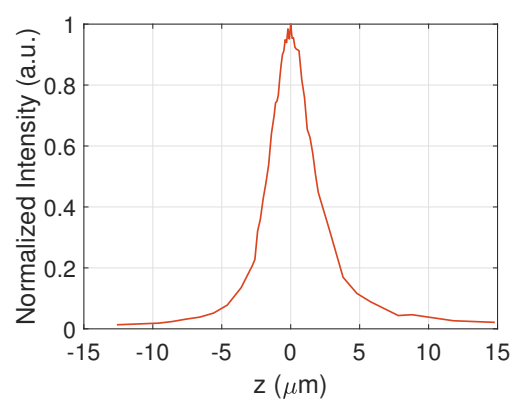

(a) Axial PSF of the $5 \times 5$ directimaging DOE.

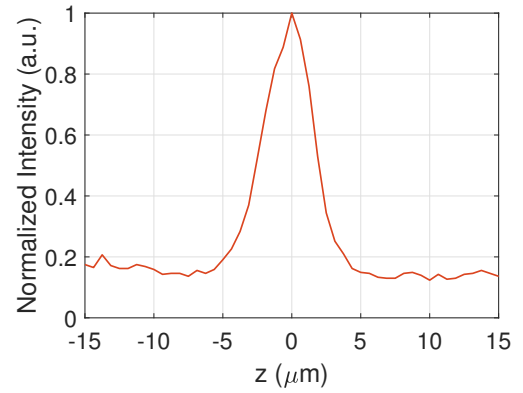

(b) Confocal axial response of the $5 \times 5$ direct-imaging DOE on a mirror.

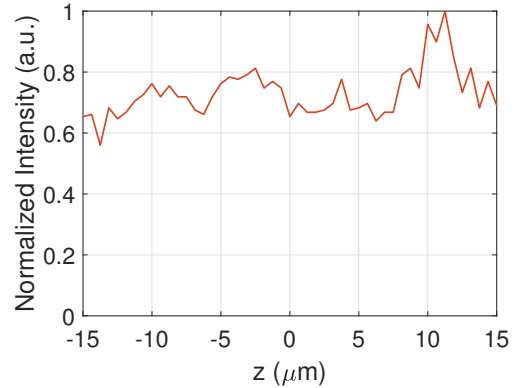

(c) Confocal axial response of the 9x9 direct-imaging DOE on a mirror.

Figure 8. Experiments to measure the confocal axial response.

\section{CONCLUSION}

In the current research, DOEs are used to replace high NA objectives in confocal microscopy to increase the field of view and reduce the cost. However, they can only measure semi-transparent samples. In this work, the ideal of superposition are proposed to overcome this limit and to enable the application of DOEs for opaque surface measurements. Two different DOE design concepts are simulated and investigated. The first see-through design can reduce the field disturbances and let the illumination spots be imaged through it. Thus it can be adapted to the confocal setup for 3D surface measurements. The second direct-imaging design has the same depth discerning capability as the traditional high-NA objectives which can provide excellent resolution.

A prototype of the DOEs is produced and tested. Experiments show that the simulation results meet the measurements very well. Both kinds of DOEs can produce highly focused spots, with an NA $=0.73$ for the see-through DOE and NA $=0.67$ for the direct-imaging DOE. However, the axial resolution of the see-through DOE is still limited by the imaging objective when a plane object is measured, although it shows a better performance measuring a rough surface. Meanwhile, the direct-imaging DOE does not have such limitations and an axial response with a FWHM of $4.57 \mu \mathrm{m}$ is measured. However, it suffers from severe field disturbances. 1D arrangement of the spots as a line is proposed to reduce the interference for real industrial applications.

In the future, experiments for the system measurement uncertainties will be conducted. A new prototype with multi-level phase structures is planned for better diffraction efficiency. Furthermore, new possibilities to combine structured illumination and interference with the DOEs will be considered for 3D surface measurements. Other new applications like fluorescence microscopy will also be investigated. 


\section{REFERENCES}

[1] Vangindertael, J., Beets, I., Rocha, S., Dedecker, P., Schoofs, L., Vanhoorelbeke, K., Hofkens, J., and Mizuno, H., "Super-resolution mapping of glutamate receptors in c. elegans by confocal correlated palm," Scientific reports 5, 13532 (2015).

[2] Zheng, G., [Fourier Ptychographic Imaging: A Matlab Tutorial], Morgan \& Claypool Publishers (2016).

[3] Hulsken, B., Vossen, D., and Stallinga, S., "High NA diffractive array illuminators and application in a multi-spot scanning microscope," Journal of the European Optical Society-Rapid publications 7 (2012).

[4] Liu, X., Stenau, T., and Brenner, K.-H., "Diffractive micro lens arrays with overlapping apertures," in [Information Optics (WIO), 2012 11th Euro-American Workshop on], 1-2, IEEE (2012).

[5] Stenau, T. and Brenner, K.-H., "Diffractive lenses with overlapping aperture a new tool in scanning microscopy," in [Imaging Systems and Applications], IT1F-1, Optical Society of America (2016).

[6] Liu, X. and Brenner, K.-H., "High resolution wavefront measurement with phase retrieval using a diffractive overlapping micro lens array," in [Fringe 2013], Osten, W., ed., 233-236, Springer Berlin Heidelberg, Berlin, Heidelberg (2014).

[7] Sommerfeld, A., "Mathematische theorie der diffraction," Mathematische Annalen 47(2), 317-374 (1896).

[8] Sommerfeld, A., [Mathematical Theory of Diffraction], Birkhäuser Boston, Boston, MA (2004).

[9] Shen, F. and Wang, A., "Fast-fourier-transform based numerical integration method for the rayleighsommerfeld diffraction formula," Applied optics 45(6), 1102-1110 (2006).

[10] Dai, E., Zhou, C., Xi, P., and Liu, L., "Multifunctional double-layered diffractive optical element," Optics letters 28(17), 1513-1515 (2003).

[11] Hell, S. W. and Wichmann, J., "Breaking the diffraction resolution limit by stimulated emission: stimulatedemission-depletion fluorescence microscopy," Optics letters 19(11), 780-782 (1994).

[12] Betzig, E., Patterson, G. H., Sougrat, R., Lindwasser, O. W., Olenych, S., Bonifacino, J. S., Davidson, M. W., Lippincott-Schwartz, J., and Hess, H. F., "Imaging intracellular fluorescent proteins at nanometer resolution," Science 313(5793), 1642-1645 (2006).

[13] Li, Z., "Application of diffractive optical elements in confocal microscopy," in [Proceedings of the 2018 Joint Workshop of Fraunhofer IOSB and Institute for Anthropomatics, Vision and Fusion Laboratory], Taphanel, M. and Beyerer, J., eds., KIT Scientific Publishing, Karlsruhe (2019).

[14] Wilson, T. and Sheppard, C., [Theory and practice of scanning optical microscopy], vol. 180, Academic Press London (1984).

[15] Born, M. and Wolf, E., [Principles of optics: electromagnetic theory of propagation, interference and diffraction of light], Elsevier (2013).

[16] Kirshner, H., Sage, D., and Unser, M., "3D PSF models for fluorescence microscopy in ImageJ," in [Proceedings of the Twelfth International Conference on Methods and Applications of Fluorescence Spectroscopy, Imaging and Probes (MAF'11)], 154 (September 11-14, 2011). 